\title{
VALPROATE-INDUCED REPRODUCTIVE DISORDERS
}

The relation of antiepileptic therapy to the occurrence of reproductive endocrine disorders in 238 women with epilepsy has been studied at the University Hospital, Oulu, Finland. Age ranged from 18 to 45 years (mean, 33 $\mathrm{yrs})$; the mean duration of therapy was 9 years. Menstrual disturbances occurring in $20 \%$ of patients were unrelated to the type of epilepsy but were significantly more frequent in those treated with valproate alone than in patients receiving other AEDs. Polycystic ovaries and elevated serum testosterone concentrations were found in the majority of women who were taking valproate and especially in those beginning treatment in adolescence; $80 \%$ receiving valproate before age 20 years were affected, compared to $27 \%$ of women treated with other AEDs. (Isojarvi JIT et al. Polycystic ovaries and hyperandrogenism in women taking valproate for epilepsy. N Engl I Med Nov 4 1993;329:1383-8). (Reprints: Dr Isojarvi, Dept of Neurology, University of Oulu, SF90220 Oulu, Finland).

COMMENT. Polycystic ovaries, hyperandrogenism, obesity, menstrual disturbances, and hirsutism, characteristic findings of the polycystic ovary syndrome, may be secondary to valproate therapy for epilepsy. Women who begin treatment in adolescence are especially at risk. The authors voice concern regarding the use of valproate in adolescent and young women with epilepsy, and alternative antiepileptic medications should be selected for female patients in these age groups when possible.

Impaired fatty acid oxidation in 12 children taking valproic acid and at low risk for hepatotoxicity ( $>2$ years of age and monotherapy) is reported from the University of Chicago (Kossak BD et al. Neurology Nov 1993; $43: 2362-8)$. The valproate-induced metabolic abnormalities resembled those found in inborn errors of mitochondrial B-oxidation and were not corrected by L-carnitine supplementation. These findings refute the hypothesis of "carnitine insufficiency" as the cause of valproate hepatotoxicity, and the routine supplements of carnitine in patients taking valproate are not advised.

\section{VON HIPPEL-LINDAU DISEASE AND PHEOCHROMOCYTOMA}

Nineteen percent of 82 unselected patients with pheochromocytoma studied at the University of Freiburg, Germany, and the University of California, San Diego, were found to be gene carriers of von Hippel-Lindau disease. Thirty-eight percent of carriers of von-Hippel-Lindau disease had pheochromocytoma as the only manifestation of their syndrome. In 12 of 14 families with von Hippel-Lindau disease, pheochromocytoma, retinal angiomatosis, and hemangioblastoma of the central nervous system occurred in the absence of renal, pancreatic, and epididymal lesions, which suggests a 
distinct subtype of the disease with unique molecular-genetic characteristics. Hemangioblastoma developed in $17 \%$ of carriers. (Neumann HPH et al. Pheochromocytomas, multiple endocrine neoplasia type 2, and von Hippel-

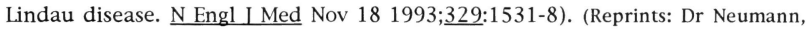
Division of Nephrology and Hypertension, University of Freiburg, Hugstetterstr. 55, D79106 Freiburg im Breisgau, Germany).

COMMENT. All patients with pheochromocytomas should be screened for von Hippel-Lindau disease by ophthalmoscopy and MRI of the brain. Retinal angiomas are usually asymptomatic. Hemangioblastoma of the CNS has an excellent prognosis if recognized and removed early.

A three-decade investigation of familial pheochromocytoma involving 619 descendants of 3 siblings of German origin, reported from the University of Pittsburgh (Tisherman SE. Arch Intern Med Nov 22 1993; 153:2550-6), showed that education and screening decreased mortality. Symptoms on presentation were the classic triad of sweating, nervousness, and headaches. Weight loss, nausea, vomiting, and abdominal pain were more common in children than in adults with pheochromocytoma. Six had von Hippel-Lindau disease manifested by cerebellar hemangioblastoma or retinal angioma, one becoming blind from bleeding. Regular screening and early diagnosis are important to prevent blindness.

\section{METABOLIC AND DEGENERATIVE DISORDERS}

\section{VITAMIN E DEFICIENCY FAMILIAL ATAXIC SYNDROME}

A total of 8 members of two consanguineous Tunisian families affected with Friedreich's ataxia (FA) phenotype not linked to chromosome 9 were found to have very low levels of serum vitamin $\mathrm{E}(0.5 \mathrm{mcg} / \mathrm{ml} \mathrm{cf} 8 \mathrm{mcg} / \mathrm{ml}$ in controls) in a study at the Institut National de Neurologie, Tunis, and at Centers in Cyprus and France. All patients had typical signs of FA: severe ataxia, bilateral Babinski signs, pes cavus, scoliosis, and absent tendon reflexes and proprioception. The mean age at onset was 11 years, and 6 patients were wheelchair-bound at examination at 20 - 30 years of age. Cardiomyopathy was found in 5. Parents and healthy sibs had normal serum vitamin E levels, pointing to an autosomal recessive trait in affected members. Cystic fibrosis, abetalipoproteinemia, and fat malabsorption syndromes were excluded as possible causes of vitamin E deficiency. This FA with selective vitamin E deficiency was mapped to chromosome 8q, confirming its specificity. (Hamida $\mathrm{MB}$ et al. Friedreich's ataxia phenotype not linked to chromosome 9 and associated with selective autosomal recessive vitamin E deficiency in two inbred Tunisian families. Neurology Nov 1993;43:2179-2183). (Reprints: Prof Mongi Ben Hamida, Institut National de Neurologie, La Rabta 1007 Tunis, Tunisia). 